forthcoming in Nô̂s, ca. 2018.

\title{
Absolute time: the limit of Kant's idealism
}

\author{
Marius Stan
}

\begin{abstract}
I examine here if Kant can explain our knowledge of duration by showing that time has metric structure. To do so, I spell out two possible solutions: time's metric could be intrinsic or extrinsic. I argue that Kant's resources are too weak to secure an intrinsic, transcendentally-based temporal metrics; but he can supply an extrinsic metric, based in a metaphysical fact about matter. I conclude that Transcendental Idealism is incomplete: it cannot account for the durative aspects of experience-or it can do so only with help from a non-trivial metaphysics of material substance. ${ }^{1}$
\end{abstract}

I have two aims here: first, to argue that Kant's idealism about time is insufficient. That is because temporal experience-objective knowledge of duration-requires time to have a metric, and yet transcendental resources cannot meet that demand on Kant's behalf. Specifically, the constitutive apparatus of his Critique is too weak to yield enough metric structure for time. Second, I aim to prove, Kant's metaphysics entails a fact (about matter) strong enough to endow time with a metric, and so to make chronometric knowledge a meaningful endeavor. Eo ipso that shows, and fills, a gap in transcendental idealism. I move now to motivate my aims, from three angles.

Early moderns before him struggled with a metaphysical conundrum about time. Some took it to be substantival: a thing-like existing independently of matter. Others countered that time $i s$ nothing over and above the temporal relations obtaining directly between material bits or processes. Kant advertised his idealism as solving this conundrum, inter alia.

\footnotetext{
${ }^{1}$ I am greatly indebted to Michael Friedman for his insightful, deep and incisive thoughts on a previous draft of this paper. I am grateful to David Hyder for detailed, helpful conversations on the topic, and to two anonymous referees for their trenchant observations. I thank Katherine Dunlop, James Messina, Bennett McNulty, Jean-Luc Solère, and Richard Atkins for their valuable suggestions and constructive advice.
} 
Time, he claimed, is neither substantival nor relational; it really is a mental structure, and any temporal feature of objects is parasitic on facts about human minds. Kant gave few details about this doctrine, and if we examine it closely it turns out that his theory of the mind lacks the resources needed to endow time with a metric. The metric comes from his metaphysics of matter, not transcendental psychology; or so I argue.

Another angle: metaphysicians in our time have debated whether time's metric is intrinsic or extrinsic. ${ }^{2}$ Kant famously claimed time to be an a priori form that precedes explanatorily both objects having chronometric properties and our knowing them. Then we might think that for Kant the metric of time must count as intrinsic. But that is mistaken, I prove below, because time-as-form lacks any intrinsic, built-in metric properties. Only Kant's metaphysics (of matter) can induce a metric on time, which thus is extrinsic for him.

Finally, Kant has internal reasons to ponder this question. The Critique set out to uncover the enabling conditions for possible experience, and time affects it at two levels. In a thin sense, experience is temporal in that any output of perceptual awareness always succeeds some other output. But experience is temporal also in a thicker, more demanding sense: we make knowledge claims about durations, pervasively and uncontroversially. ${ }^{3}$ In Kant's terms, the concept of duration has "objective reality." But what is the Kantian ground for that presumption? To see the problem clearly, ask yourself: what makes it the case that two time spans are equal? What sets the time that any clock keeps qua timekeeper? Are there Kantian facts about how long events really last? What are they?

We are tempted to answer: mental facts. That is, facts about time as a mental structure; or about some "pure synthesis," a non-empirical product of mental activity. Inviting as this may look, it is ultimately wrong, I argue. In Kant's system, mere features of the mind cannot ground chro-

\footnotetext{
${ }^{2}$ There are two approaches to this problem. Grünbaum 1970 and Glymour 1972 start from definability in set-theoretical terms as a criterion for intrinsic structure. Bricker 1993 and Skow 2010 analyze intrinsic/extrinsic as entrenched in Lewisian metaphysics.

${ }^{3}$ Though we may debate about the exact time length of this or that event, no one disputes that "length of time interval" is a legitimate, well-defined concept (at least in a classical regime). Even in Special Relativity duration is objective, though frame-dependent: for any inertial observer $O$, there is a fact about the time-length of events observable in $O$ 's frame.
} 
nometry. Rather, time has a metric in virtue of a nomic fact about matter: a universal necessary fact about kinematic relations between material volumes. I call it Kant's Area Law, and explain it below.

My study uncovers a type of ground, a central notion in both Kant and analytic metaphysics; and so the latter, used wisely, can be a useful lens for my topic. Kantian grounds come in two species, transcendental and metaphysical. The former are mind-inherent enablers for certain cognitive achievements; the latter amount to relations of ontological dependence. From this viewpoint, I claim, the Kantian ground of time's metric is metaphysical, not transcendental-initial expectations to the contrary. And, it is a determining ground: it explains why any specific duration has the time-length that it does; and is the final evidence (and truth-maker) for claims about specific durations. ${ }^{4}$ Some grounds are intrinsic, others are extrinsic. The epistemic criterion for ascertaining intrinsic grounding is definability: if we can define T's metric properties wholly in terms of facts about $\mathrm{T}$ itself, then T's metric is intrinsic. In consequence, Kant's possible sources of grounding are these.

F-intrinsic: chronometric relations depend directly on points of time as a sensible form. They are fundamental. D-intrinsic: congruence is defined on time instants, but in terms of more basic, non-metric facts about time, which ground it. Chronometric relations are derivative. U-extrinsic: congruence is induced on time intervals by a priori structures of the understanding, viz. categories. S-extrinsic: time congruence is parasitic on relations between the points of nonempirical Kantian space. M-extrinsic: time congruence depends on relations between material points.

I claim that Kant's considered view is an M-extrinsic account.

I draw three lessons from my results, one for each audience above. $i$. Inspecting Kant's full theory of time reveals that he was a relationist. Despite his advertising, he did not offer a real third way beyond Newton and Leibniz. $i i$. In Kant, time's metric is extrinsic, qua parasitic on a nomic fact about matter. iii. To live up to its explanatory promise, transcendental idealism needs help from a non-trivial ontology of material substance.

\footnotetext{
${ }^{4}$ Projects to connect Kant's doctrine with contemporary accounts of ground started with Smit 2009; cf. also the helpful accounts in Massimi 2017 and Messina 2017.
} 
Against this backdrop, my argument is in four parts. Part I is a preamble that explains the problem and its implications. Part II distinguishes two key aspects of time, topological and metric, and then explains how they bear on Kant. Part III subverts existing alternatives to my solution. Part IV gives my solution, and draws some conclusions. I give an informal deductive proof of Kant's Area Law, from his premises (Appendix).

Before I set out, a brief note on method. My account is reconstructive: I aim for an analytic explication, not a textual interpretation-and so I put my points both in Kant's terms and in ours, for greater precision. Still, to avoid anachronism and keep some interpretive value for my paper, I rely just on insights (in principle) available to Kant, and I contextualize them to his time.

Part I This part motivates my study. Below, I clarify Kant's problem; then I point out the cost of leaving it unsolved, and I spell out conditions of adequacy for a solution (Sec. 2).

\section{Kant's problem, explicated}

I am asking about Kant's grounds of knowledge of determinate duration, or quantitative knowledge about the length of time intervals. Given his project, such knowledge is unavailable unless he can show time to have a metric.

Intuitively, a metric is a set of facts about distances between points, and about the length of intervals having them as endpoints. These facts ground objective answers to questions such as: How big is it? How long did it last? How far was it? More exactly, a metric is a system of dyadic relations between any two points in a manifold, continuous or discrete, such that to each relation a number is associated. Mathematics expresses this idea by means of two related but distinct notions: distance functions, mapping end-points of intervals to non-negative real numbers; and measure functions, mapping finite curves, surfaces and volumes to numbers. ${ }^{5}$

\footnotetext{
${ }^{5}$ A distance is any function $\mathbf{d}$ satisfying three conditions: 1 . For any two points $\mathrm{F}, \mathrm{G}, \mathbf{d}_{F G}$ is positive; and zero iff $\mathrm{F}=\mathrm{G}$. 2. Symmetry. 3. The 'triangle inequality,' $\mathbf{d}_{F G}+\mathbf{d}_{G H} \geq \mathbf{d}_{F H}$.
} 
Then we may ask Kant a crucial question: what facts ground time having such numeric properties? Are they facts about time alone? Or are they about things and structures other than time? Metaphysics now puts it as the question whether temporal metric is intrinsic or extrinsic.

Start with the notion of a manifold $T$ of instants, or points of time. Consider a set of relations $D_{i j}$ defined for any two points $i, j$ in $T$, such that a distance function $\mathbf{d}$ will map any $D_{i j}$ into some real number. That makes every $D_{i j}$ a species of a relational property $D$, or duration. Now $D$ has two possible ontologies. Intrinsic: the temporal metric $D$ counts as intrinsic just in case its obtaining depends on entities "internal" to $T$, viz. on instants as its members. Namely, $T$ would have the relations $D_{i j}$ regardless of "whether or not there were people, languages, automata or anything" other than the manifold itself. Extrinsic: $D$ depends for its obtaining on entities external to $T$, in the sense of "non-internal." Candidate entities are space intervals; or physical systems, whether natural or manmade clocks. ${ }^{6}$

I argue that Kant should think that time's metric is extrinsic. Namely, that all material points stand in certain relations that can endow time with a congruence structure; and no other source of metric can do so.

Kant was familiar with this problem, though I cannot tell how clearly he saw its difficulty. In a famous paper known to him, Euler had argued for substantival time, against relationism. "Consider a body that crosses equal spaces in equal times: by what changes or by what reference body must we judge the equality of these times?," he asked. He then reviewed two relationist replies, showed their inadequacy, and concluded: only by having time "subsist outside our mind as something real" can we ground temporal congruence (Euler 1750: $\S 21$ ). So, the ontology of time's metric was a live issue in Kant's age.

Then what is his metaphysics of chronometry, and what entitles him to it? Unfortunately, on this count Kant lacks a clear answer and an explicit case for it, so I will make it on his behalf. Put clearly, we expect that

In turn, a measure $\mathbf{m}$ is an assignment of size (for a set $\mathrm{A}$ with subsets $\mathrm{A}_{i}$ ) defined by three axioms: (1') Non-negativity: $\mathbf{m}\left(\mathrm{A}_{i}\right) \geq 0$. (2') Null measure: for any singleton set $\mathrm{L}$ in $\mathrm{A}$, $\mathbf{m}(\mathrm{L})=0$. (3') Additivity: if $\mathrm{A}_{i}$ are disjoint sets, $\mathbf{m}\left(\mathbf{U} \mathrm{A}_{i}\right)=\sum \mathbf{m}\left(\mathrm{A}_{i}\right)$; $\mathbf{U}$ denotes settheoretic union and $\Sigma$ is Riemann summation.

${ }^{6}$ See Grünbaum (1970: 525ff) and Glymour (1972: 326). 
for any event it makes sense to ask if it took as long as some other event; and whether time passes equably or not. But these questions are meaningful just in case two theses are true:

Congruence: there are facts in virtue of which time intervals are equal, or congruent. Steadiness: there are facts in virtue of which time passes at a constant rate, or equably; thus it does not speed up or slow down. ${ }^{7}$

The problem is that Kant has no overt account of Congruence and Steadiness. I claim that he could establish both. Now here is why he must.

\section{Kantian grounds for metrics; necessity}

Time is a key load-bearing pillar in Kant's mature system, which arose largely out of a need to vindicate quantitative knowledge, thus he needs a sound foundation for chronometry. And, given how much he staked on temporality, Kant needs a foundation that is in some sense necessary; a contingent grounding for time's metric would ruin his entire Critical project. Consider:

In Foundations, Kant set out to anchor mathematical physics in an ontology obtained by applying his categories to the concept of matter. The applying was a sophisticated blend of conceptual analysis, transcendental argument and 'construction,' or a priori generation of singular representations with universal probative force. Kant's first construction was the 'composition of motions.' Namely, he proved that any two (instantaneous linear) velocities of a movable point add up (vectorially) to the diagonal of the parallelogram they form with each other. ${ }^{8}$ By his criteria this addition rule is synthetic a priori, so Kant constructs it in pure intuition, to show that it is apodictic and universal. Consider the case in which the two component velocities are at an angle (and the resultant is the diagonal):

\footnotetext{
${ }^{7}$ Contemporary metaphysics puts this idea in terms of time passing at the rate of one second per second. See Maudlin 2007 and Skow 2011 for discussion.

${ }^{8}$ 4: 486-94; see explanations in Friedman (2013: 67-75), Hyder 2013, Sutherland 2014.
} 


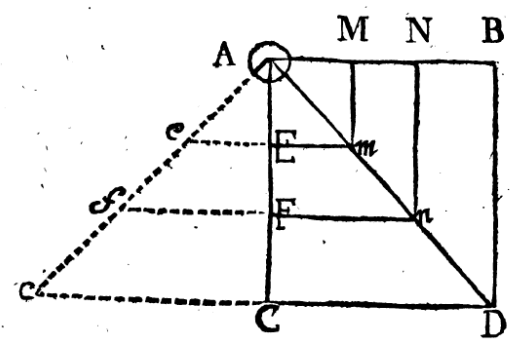

[Fig. 1. Kant's a priori construction of the 'composition of motions.' If a mobile point $A$ has, or is given, two simultaneous linear velocities proportional to $A B$ and $A C$ respectively, then $A$ will move with a resultant equal in speed to $A D$, along the diagonal of $A B D C$, a (rectangular) parallelogram. This is a particular case-for velocities at right angles - of the Parallelogram Rule for adding oriented magnitudes, in early modern science. Kant 1794: 22.]

Note a fact that Kant chose to keep tacit. His very construction above makes clear that he must assume both Congruence and Steadiness. Specifically, the segments AM, MN, NB are mutually congruent, and stand for equal times elapsed consecutively as the point crosses the finite path $\mathrm{AB}$. (So do $\mathrm{AE}, \mathrm{EF}, \mathrm{FC}$; $\mathrm{A} m, m n, n \mathrm{D}$; and $\mathrm{A} e, e f, f c$ respectively.) But, his assumption - that equal line segments may stand in for equal times - is legitimate only if time has in fact metric structure, such that Congruence and Steadiness obtain. Without them, Kant's proof of the Composition of Motion collapses, with disastrous domino effects: his entire Foundations rests on the claim that motions compose according to his Parallelogram Rule above. Simply put, without an argument for temporal metric Kant cannot afford his natural philosophy.

Second, whether time's metric is intrinsic would cast much needed light on a notably dark recess in the Critique. Though Kant fully reworked in B the Transcendental Aesthetic's presentation makeup, time still got short shrift: it has no real analogue of the 'argument from geometry.' Instead of a lucid account, Kant gestured vaguely at allgemeine Bewegungslehre, a mysterious designation. ${ }^{9}$ And so, we still do not know what sci-

\footnotetext{
${ }^{9}$ Perhaps by 'general doctrine of motion' he means his Phoronomy in Foundations. Still, Kant there describes Phoronomy as "pure doctrine of motion," to distinguish it from the "applied" part of the general doctrine. And, before and after the Critique he had claimed that time-as-form is presupposed in "pure mechanics," clearly a broader theory than the 1-
} 
ence requires indispensably that time be nothing but the form of inner sense, and how much structure it has. Without a clear account of Kantian chronometry, it is unclear what a Transcendental Exposition of the Concept of Time achieves.

In fact, the issue of chronometric structure percolates into the BDeduction and Schematism, the very heart of Kant's system. The categories of quantity have a joint schema, viz. "number." And, time is the official medium in which the categories must be schematized to yield concepts of an object. This suggests chronometric relations are U-extrinsic, induced by quantity-categories. Yet his claim remains stubbornly opaque, because applying number to a sensible manifold amounts to countingbut time is continuous, and a continuous manifold is uncountably dense, a fact known since Leibniz. So, either Kant presumes to count the innumerable (viz. the set of instants in any time span) or number is not the schema of quantity. Then what is?

To baffle the reader further, Kant had claimed (in the B Deduction) that categories apply to sensibility by always directing some figurative synthesis, speciosa. His explanation is: "We cannot represent time itself without attending, in the drawing of a straight line (which is to be the outer figurative representation of time), merely to the action of synthesis of the manifold, through which we successively determine inner sense, and thereby attend to the succession of this determination in it" (B 154). This implies that chronometric structure after all is S-extrinsic, not U-extrinsic. Namely, $\S 24$ entails that representing time is indirect thinking: one must intuit a 'pure' line (hence auto-generated by the very mind that intuits it) and let that line stand for time. Which entails that metric relations on the Euclidean line ground temporal congruence, and duration generally.

Conditions of adequacy. Other scholars have addressed Kant's problem before me, and no doubt more will emerge. To avert unmanageable

point kinematics he calls 'Phoronomy' (cf. Inaugural Dissertation $\S 12$; Prolegomena $\S 10$ ). Falkenstein follows him, and takes time to ground "the whole of mechanics" (1995: 271). Confusingly, Kant also connects time to arithmetic; which he sanctioned in Schulz' explication of the Critique: space and time being pure intuitions "makes clear how geometry and arithmetic-i.e. pure mathematics-is possible" (Schulz 1784: 24). This led some to doubt that Kant could connect his time-form to any a priori discipline; cf. Kemp Smith (1967: 137) and Melnick (1989: 24). 
proliferation and help select the best account, I list three constraints on solutions. Necessity: a solution should entail that Kant's foundations of chronometry are in some sense necessary. ${ }^{10}$ This constraint is nonnegotiable. As I explained above, key parts of Kant's system (which is all necessary knowledge) depend explanatorily on time having a metric, and so a solution making chronometry contingent would wreak havoc to his system. Coverage: a proper solution must cover all of time, by inducing metric structure on all temporally-ordered objects and processes to which humans have epistemic access. It must not entail that events exist for which there is no fact about how long they last. Exactness: an account of chronometric structure in Kant must be exact, i.e. ground arbitrarily precise time measurements (modulo technological limits on measuring operations). This is really two criteria. The account should entail that, for any given time interval, there is a determinate fact about its duration. And, it must entail that any sequence of increasingly precise measurement will converge toward a single determinate value.

Part II To solve Kant's problem, I distinguish two kinds of temporal structure, viz. topological and metric; in turn, metric facts come in two species. Then I adapt these distinctions to Kant's doctrine, in Sec. 4.

\section{Analytic tools: topology vs metric(s)}

At this juncture I must introduce two distinctions. The first is indispensable-without it, Kant's problem is not even visible, let alone solvable. The second is crucial for a correct solution. Once I explicate them, both my solution and also the defects in previous readings become easier to grasp.

The first distinction is between topology and metric. ${ }^{11}$ Intuitively, it is the difference between properties pertaining to serial order of situation and those pertaining to size in the most general sense. The main topologi-

\footnotetext{
${ }^{10}$ Necessity comes in several kinds in Kant's doctrine, so, depending on the specific necessity implied by a particular solution, its ramifications will need careful scrutiny. Stang 2016 analyzes lucidly two kinds of necessity in Kant.

${ }^{11}$ The demand to distinguish topological from metric aspects of space and time goes back to Reichenbach. Glymour 1972 and Friedman 1983 follow him in that regard, and so do I.
} 
cal features of a manifold are: dimensionality; orientability, i.e. allowing distinctions between left and right; continuity or discreteness; connectedness, or whether any two points can be joined by an 'unbroken,' connected path; and compactness. In contrast, metric properties are distance; curvature and torsion; and also length. Topological and metric facts differ in kind, for they vary independently: two manifolds can have the same topology but different metrics, and vice-versa. ${ }^{12}$

And, we may divide metric aspects into two classes: distance relations, and length properties. For two reasons, we must pry them apart. $i$ ) Even for straight lines, length is the natural concept, being the onedimensional (1D) version of a truly general notion, viz. size. Distance is an ad-hoc species without a genus-a historical relic used (for lack of better options) before the advent of measure theory. $i$ ) Because of its premodern origin, distance-then defined as a ratio to some finite unit segment-is inextricably tied to classical notions of mathematical existence, namely to constructability by compass and straightedge. That makes distance-concepts significantly limited, and creates a serious problem for Kant. Only by avoiding the notion of time distance entirely (as I do) can we extricate Kant from his troubles with chronometry.

To see the distinction, take a segment PQ of a parabola. Distance associates a number with the straight line segment having $P$ and $Q$ as endpoints. Length is the measure of PQ taken along the parabolic curve. Now consider exact expressions of these ideas. Let $G, H$ be points on the real line $\mathbf{R}$ and $\mathbf{m}$ be a measure. The distance $\mathbf{d}$ between the two points is:

$$
\mathbf{d}_{G H}=|y-x|
$$

where $x, \mathrm{y}$ are their respective coordinates (expressed in real numbers); and the length 1 of the interval GH having them as endpoints is: ${ }^{13}$

\footnotetext{
${ }^{12}$ For an intuitive example, consider a set of people. We may order them differently (e.g. alphabetically, by weight, by height) while keeping their mutual distances the same. And we can change their mutual distances while one such order (their combinatorial topology) stays fixed.

${ }^{13} e$ is a point in a manifold $M^{n}$ of $n$ dimensions, and $d e$ an element of integration. A measure assigns a size to every de, enabling the whole to have a size; cf. Lebesgue 1927.
} 


$$
\mathbf{1}_{G H}=\int_{G}{ }^{H} \mathbf{m}(e) d e
$$

On the real line, these two properties happen to be ratio-preserving. ${ }^{14}$ That happy accident might suggest they are interchangeable; but they are wholly distinct properties, and in general they do not preserve the same ratios. The reason is that line-distance generalizes as $n$-dimensional distance, whereas length generalizes as size, or measure. These two metric notions thus come apart in all manifolds but one. That distance and length track each other's behavior on the real line (by preserving ratios) must not seduce us into confusing them. They are distinct, and so we must distinguish them as we seek a solution to Kant's problem.

In discrete manifolds, both properties above are intrinsic. Start with the notion of measure and the plausible assumption that cardinality ('card') gives a natural measure. ${ }^{15}$ In plain terms, the size of a discrete interval $X$ equals the number of elements in it:

$$
\mathbf{m}(X)=\operatorname{card}(X)
$$

Define the distance between two endpoints A, B of a discrete interval as:

$$
\mathbf{d}_{A B}=\mathbf{m}(\{x: \mathrm{A} \leq x \leq \mathrm{B}\})-1
$$

In words: the distance from $A$ to $B$ equals the number of elements from one end to the other. (E.g. there are seven days from one Monday to the next.) Now cardinality is an internal property: it depends on nothing except the elements that make up an interval. Since measure and distance are wholly definable in terms of cardinality, they count as intrinsic (van Fraassen 1969).

In contrast, a continuous manifold has no intrinsic metric; essentially, it is because such manifolds are uncountably dense. ${ }^{16}$ Hence modern

\footnotetext{
14 'Ratio-preserving' means that, for any two segments GH, JK, the ratio of their length $\mathbf{1}_{G H} / \mathbf{1}_{J K}$ always equals the ratio $\mathbf{d}_{G H} / \mathbf{d}_{J K}$ of the distance between their respective endpoints.

${ }_{1}$ Warning: though it appears natural, it really is degenerate. This measure violates axiom $(2 ')$ of measure theory, viz. that all unit sets have measure zero. See note 3.

${ }^{16}$ The continuum being uncountable, cardinality is not a natural measure for the length of intervals. (All continuum intervals have the same cardinality, even those of different
} 
authors have concluded that, in any continuum, metric facts are extrinsic. Specifically, two continuous intervals $A B, C D$ count as congruent-in the sense of having respectively equidistant endpoints-just in case the same rigid rod can be superimposed over them consecutively. Congruence in continua is an indirect relation between intervals, qua mediated by transportable material objects, hence dependent on their existence and makeup. ${ }^{17}$ So, congruence relations are extrinsic, and so are the metric facts they entail.

Context. These distinctions are not anachronistic for Kant, only their exact statement is. His age grasped them too, though just intuitively. Clarke and Newton knew that serial order (i.e. topology) differs from metric properties, and used it to attack Leibniz's anti-substantivalism about time: "going before, and following, constitute situation or order: but the distance, interval, or quantity of time or space, wherein one thing follows another, is entirely a distinct thing from the situation or order, and does not constitute any quantity of situation or order." They are distinct, as metric facts can vary while topological ones do not: "the situation or order may be the same, when the quantity of time or space intervening is very different," Clarke explained (Alexander 1970: 105; my italics). Euler too saw that order of situation is distinct from metric aspects, and can be studied without them. In geometria situs, as he called it, we "neither need attend to quantities nor employ any calculus of quantities," he explained as he went on to solve the first problem in topology (Euler 1736: 128). In Kant's time, Hindenburg defined distance in terms of place in a serial order, just as expression (3.4) above has it: "Absolute position-of parts relative to the whole-regards the number of places and the distance whereby a part is removed from both end-places" of a combinatorial series. He credited Leibniz for the insight (Hindenburg 1781: v). Even (3.2) above had antecedents in Kant's time. In a textbook, two famous Newtonians

length.) Lacking a preferred measure, any assignment $\mathbf{m}, \mathbf{m}^{\prime}, \mathbf{m}^{\prime \prime}$ of size will do just in case it meets the conditions on a measure function. But, non-trivially different measures allow non-trivially different distance functions $\mathbf{d}, \mathbf{d}^{\prime}, \mathbf{d}^{\prime \prime}$ to be defined from them. That makes the continuum metrically amorphous: alternative metrics can be defined from its elements, and no such metric is distinguished over the others. See details in van Fraassen 1969.

${ }^{17}$ In particular, the rod must be rigid, so that it necessarily remains self-congruent throughout its transport from one interval of the continuum to another. 
explained: "We denote as $\int d x$ the integral of a differential $d x$; for we consider $d x$ to be an element of its integral $x$, which integral we think as the sum of its elements $d x "$ (LeSeur \& Jacquier 1768: 4). In the Enlightenment, size-or extensive magnitude in space, as Kant put it-came in species, based on dimension: "rectification" denoted length, or line integral; "quadrature" was area, or surface integral; and "cubature" was their name for the size of volumes (see, e.g. Agnesi 1748: 709; Sauri 1778: 61-101; Cousin 1777: §§ 183ff; Lacroix 1796: 189).

Even Kant saw the first distinction above, but dimly, as he announced his discovery of incongruent counterparts. Kant could not express it yet, but his insight was topological: into chirality (or handedness), a non-metric property that only orientable spaces have. ${ }^{18}$ And, he had an intuitive grasp of key topological notions, e.g. dimension, continuous, discrete, and partition into intervals. Because of his constructivist commitments, however, Kant relied on certain preferred manifolds and their topologies to represent these notions. In particular, for him space and time typified continuity and dimension, while the natural numbers were the discrete manifold par excellence.

And so, here is how my distinctions above translate to the specific case of time, and thereby to Kant's problem. The following are topological, not metric facts. Time is a serial order of situation, and 〈later than $\rangle$ is a complete order relation between instants. Time has just one dimension. Time is continuous, not discrete nor denumerably dense. Time is connected, or 'unbroken.'

And, these facts are metric, not topological. Duration: temporal length or distance is well defined, and time has a complete partition into equivalence classes of equal time intervals. Passage: time has a determinate rate of change at every instant (namely, zero) hence Steadiness is a fact. Curvature and torsion: both are trivially zero everywhere on the time-line.

I made these distinctions so as to raise three questions essential to making sense of Kant. Which structures in his theory of time are metric, and which are just topological? What concept of duration can we get from

\footnotetext{
${ }^{18} \mathrm{Kant}$ 's insight is non-metric,as he argues that counterparthood is irreducible to relations of congruence, equality and similarity, which are metric. Some bodies have "true differences" irreducible to difference in "shape" or "magnitude of extensions" (2: 382f).
} 
him-is it distance between instants or length of time intervals? And, is he in a position to argue that time's metric is intrinsic?

\section{A problematic implication for Kant}

Daniel Sutherland has shown conclusively that Kant, for reasons crucial to his project, yoked his philosophy to a tradition of mathematics born in Euclid's work (2004). That tradition includes three built-in commitments. One is a constraint on reasoning and theory-building: the restriction to two canonical tools, viz. straightedge and compass constructions. The second is a corollary imposition: except for three objects, all existence claims must be established via canonical constructions. ${ }^{19}$ The third is a geometric algebra (credited to Eudoxus of Cnidos) that conceives of quantity as a ratio-to some finite magnitude chosen as a unit. Together these assumptions entail that $(a)$ any existence claim about particular magnitudes must be vindicated by a possible construction, viz. an iterated copying or division of the unit magnitude. And, $(b)$ only canonical tools can secure evidence for such quantity-existence claims.

Now here is the implication for Kant. Space is a continuum, so representing a specific distance $n$ amounts to constructing a line segment standing in a $n: 1$ ratio to some unit segment. But consider this task: to represent a distance of $\pi$ units. The task is impossible with Kant's Euclidean resources. ${ }^{20}$ His mathematical framework allows only compass-andstraightedge constructions, but segments of length $\pi$ cannot be so generated. In fact, the problem is even more severe, for $\pi$ is not the only number unconstructable geometrically. There are others, e.g. Euler's number $e$. Lambert and Euler knew that $\pi$ and $e$ are 'transcendent,' in the sense that no segment equal to their respective length can be produced by straightedge-and-compass operations. But there is an infinity of transcendent

\footnotetext{
${ }^{19}$ The three exceptions are the primitive notions-point, straight line, circle-whose existence is guaranteed by the (self-certifying) Postulates I-III.

${ }^{20}$ This is a corollary of the problem of 'squaring the circle,' the impossibility of constructing (by Euclidean tools) a rectangular area $\mathrm{S}$ equal to the area $\mathrm{C}$ of a circle of given radius $r$. (If S were constructable, a segment of length $\pi$ would be too, as the geometric mean of $r^{2}$ and the sides $a$ and $b$ of S. I.e., $\pi=a b / r^{2}$.)
} 
numbers, as some knew well (Lambert 1768; Euler 1768: 13, Corollary 3; Wantzel 1837; Liouville 1844). This has a sobering consequence:

Sub-coverage. Infinitely many numbers are not Kant-constructible in pure intuition. So, the existence of transcendent quantities cannot be secured a priori. ${ }^{21}$

Plainly put, the real line qua metric manifold cannot be assembled from parts through the constructive acts that Kant had sanctioned as the method of geometry. ${ }^{22}$ So, while we can adapt the distinction topological vs. metric reasonably well to Kant's framework, the cost is Sub-coverage.

The lesson is that Kant faces a painful trilemma. Either he gives up his philosophy of mathematics qua constructive activity; but that would sink his Critical project. Or he renounces the view that time is a continuum. Or he admits that, in an important sense, space does not have enough a priori metric structure. I choose for him the latter-it is the least damaging option.

Part III This part is an apagogic argument: I defend my solution indirectly, by showing that its competitors fail. I argue in Sec. 5 that transcendental-ideal sources of structure cannot ground chronometry; and in Sec. 6 I show that current $\mathrm{M}$-extrinsic accounts are likewise unworkable.

\section{Improper solutions, I}

Current metaphysics distinguishes two aspects: the reality of chronometric structure, no matter who knows it; and epistemic agents coming to know that time has it. ${ }^{23}$ But Transcendental Idealism rejects that distinction as

\footnotetext{
${ }^{21}$ Transcendent quantities were those impossible to generate by the canonical Euclidean algorithm, viz. a finite series of straightedge-and-compass constructions. These are not to be confused with transcendental numbers, which are the set of real numbers that are not algebraic, viz. are not solutions of polynomial equations with real coefficients.

${ }^{22}$ Modern theory avoids this shortcoming by bluntly postulating the facts that Kant demanded to see constructed. In particular, the existence of these (unconstructable) magnitudes follows from Dedekind's Axiom; they are a part of the class of Dedekind cuts.

${ }^{23}$ Trying to measure time makes sense only if time has metric properties at all-or else telling time amounts to making $u p$ facts about duration, not discovering them; and representing time as a magnitude is legitimate only if time does have a metric; cf. Massey 1970 and Skow 2010.
} 
illegitimate; Kant would counter that time having a congruence structure is inseparable from "the condition being fulfilled for our knowing" that fact in a canonical way, e.g. by a converging series of theory-mediated measurements. ${ }^{24}$ Then his inseparability claim seems to imply that there are two avenues for filling his chronometric gap, and only two. Objectivity: Metric structure is a fact about objective representations of durations. Measure: metric structure is a transcendental condition of time measurement. Now couple either option with Necessity, the key adequacy condition from section 3 above. Then, qua necessary and universal fact about time-representings, chronometric structure must be a priori, hence mindinduced. Compelling as this seems, it is inconclusive, or so I argue next. I refer the reader to Section 1, where I defined the five species of extrinsic and intrinsic metrics.

Intrinsic metrics If time is "nothing but the form of inner sense," the strong presumption is that, as form, it has metric structure a priori, independent of experience-hence intrinsic. And yet the Critique defies that presumption, if we inspect it with the tools I produced above. Kant's time-form has no direct metric relations between instants; he describes it just as an ordering of states qua successive, forming a series (B 46). So do his exegetes, for whom succession is the sole relation built a priori into time as explicated by the Transcendental Aesthetic. ${ }^{25}$ But succession turns a manifold into a serial order of situation, not a thing with size. Qua form of inner sense, time is just a topological structure, so it cannot ground any chronometric knowledge. ${ }^{26}$ Ergo, time's metric is not Fintrinsic: no basic relations of distance or length obtain on time as a sensible form.

And, it is not D-intrinsic either. Recall from $\S 4$ that, in certain manifolds, cardinality induces a natural metric: any interval (of elements in

\footnotetext{
${ }^{24}$ Cf. Parsons (2012: 61), who makes this point about Kant's conception of number.

${ }^{25}$ E.g. Valaris (2008: 13); Sutherland (2005: 150); Van Cleve (1999: 58).

${ }^{26}$ I.e. it just orders mental states (and their contents, indirectly) as successive, by the relation 〈later than〉 obtaining between any such states in virtue of time being the form of sense. But any mental state has a length (or duration) independent of its place in the time order. I.e. the contents of inner sense have metric aspects not captured by topological facts about succession.
} 
mere topological relations of nextness) can be assigned a unique length based on the number of elements; then two intervals count as congruent if their lengths are equal. Thereby congruence is derivative qua definable in terms of a more basic, non-metric relation, viz. cardinality; and yet it is intrinsic in that congruence obtains irrespective of facts, entities and processes external to the manifold itself. Could we not credit Kant with this option, so that time is the a priori form of durative experience after all? Unfortunately, we cannot. This path is closed, because D-intrinsic metrics obtain just in discrete or denumerably dense manifolds-but time is neither. It is continuous, and he knows it: "space and time are quanta continua" (B 211f). As continuous, time is uncountably dense: no instant has a unique successor, and no finite number can count the instants in a finite time interval.

Two other facts support the thesis that time-as-form has no metric. One is direct evidence; in explaining its structure, Kant lists just topological traits:

We represent the time sequence by a line going to infinity; a line in which the manifold makes up a series of just one dimension. And we infer from the properties of this line to all the properties of time; except that the parts of the former are simultaneous, but those of the latter are always successive. (B 50)

Second, Kant would have known-from du Châtelet and Euler, whom he had read-that evidence from inner sense (whose form is time) cannot determine metric facts, e.g. true durations or the equality of two times. ${ }^{27}$ If anything, inner experience speaks against Steadiness, not for it; recall the phrases, 'Time flies when you're in love' or 'That meeting lasted for ever.'

In sum, time as a sensible form lacks metric structure. Still, idealism about chronometry is not a cause perdue yet. Let us see its next move.

\footnotetext{
${ }^{27}$ In Institutions de physique $\S 112$, du Châtelet rejects Newton's absolute time but wonders what could secure time's metric, seeing as inner sense cannot: "the succession of ideas cannot serve as a means for us to help others grasp what we mean by 'such and such a portion of Time.' For, ideas succeed one another faster or slower, in different heads" (1740: 126). Euler too, in Reflections sur l'espace et le tems, rejected inner sense as a basis for chronometry (1750: $§ 20-1)$.
} 
Kant time is induced from the outside, by the understanding. They start from his words that, considered on its own (as just a manifold of instants) time is indeterminate: inner sense contains just the

mere form of intuition, yet without combination of the manifold in it; so, it contains no determinate intuition... The latter is possible only through consciousness of a manifold's being determined by the transcendental action of imagination. I.e. through the synthetic influence of the understanding on inner sense. (B 154)

On this reading, space and time being indeterminate amounts to lacking metric structure. Absent the action of the understanding, there is no fact of the matter about the size of their parts. So, metric facts are created, not given; they are brought about by the transcendental understanding, via the act of representing determinate 'spaces' and 'times,' i.e. specific lengths, distances, areas and durations. The act is a figurative synthesis: the mind generates a manifold then grasps it as one-a sort of mind drawing [zeichen], Kant says. Three aspects separate the representing of determinate spaces and times from other syntheses. First, it produces (in pure intuition) a manifold of mathematically homogeneous elements. Second, the three categories of quantity govern the constructing and its result: the self-intuited manifold is conceived as a determinate totality of 'units' standing to each other in ratios. Third, the categories are schematized-as number, the "pure schema" of magnitude (B 182). So, using categories to determine 'times' amounts to applying number-concepts, viz. to counting; thereby the intuition represents some magnitude, or quantum. In that role, it has metric properties but it acquires them from the (schematized) categories of quantity, which induce metric structure on any figurative synthesis they effect. Ergo, any (represented) particular duration has a metric aspect, extrinsically grounded in facts about the magnitude categories. ${ }^{28}$

Plausible as they seem, these construals founder once we ask: what is being counted as time determination? To what exactly are quantity-

\footnotetext{
${ }^{28}$ This reading is explicit in Longuenesse 1998, and implied by Hyder 2013. In addition, there is also Sutherland, who relies on "parts of time" in (2005: 144). However, his parts are just mereological, and so have no metric structure, thus cannot make up quantities by aggregation.
} 
categories applied so as to yield representations of particular durations? Kant says little on this topic, and so do proponents of U-extrinsic readings. To make up for their silence, I list below three candidates:

1. Points, or point-sized elements.

2. Finite-sized units $(M a \beta)$ of time, arbitrarily chosen.

3. Moments, i.e. infinitesimal increments.

I argue that none will do. 1. There is no counting the points in any finite interval of a continuous manifold. They exceed the size of the natural number series; that makes the continuum unzählbar, uncountable. ${ }^{29}$ Even if we could count them (suppose time was denumerably dense, not continuous) still no account of duration is at hand. Time-length requires a measure function-an assignment of size to its elements-and the measure of any singleton point set is zero. Hence every time span, if regarded as a denumerable union of time points, would trivially have duration zero. 2. Units will not do either. Counting finite units allows Kant to represent just integer multiples, i.e. whole-number durations. ${ }^{30}$ But time's metric must be much more fine-grained than that. In consequence, this reading fails Exactness, a condition of adequacy. 3. This seems to be the favorite construal, but exegetes just hint at it, so I spell it out here to reveal its defects. ${ }^{31}$ In Kant's time, moments were infinitesimal increments; modernly speaking, a moment is the derivative of a function at a point. Two stretches of time count as congruent, in the sense of being the same length, if they comprise the same number of moments. Generally, two stretches are proportional to the respective numbers of moments in them. So the quantity categories, schematized as number, direct figurative syntheses by having the transcendental imagination generate as many moments as required to represent specific durations.

\footnotetext{
${ }^{29}$ Cantor 1874 first proved the non-denumerability of the reals. The early moderns knew (but could not prove it) that points in a finite continuous interval cannot be counted.

${ }^{30}$ Kant knows it, and his classroom examples reflect this inadequacy: "the earth has 5400 miles--the mile is $1 / 15$ of a degree--degree $1 / 360$ of the largest meridian" (29: 992, translated in Dunlop 2009: 18, fn. 51).

${ }^{31}$ Hyder relies on "parts of time (moments)" (2013: 258); Longuenesse on "infinitesimal increases" (1998: 269). Kant's age called them synonymously 'moments,' 'infinitesimals,' 'fluxions,' or 'differences,' to reflect diverging views on the "metaphysics of the calculus," i.e. the composition of the continuum (Grabiner 2011).
} 
This account is fatally flawed. Moments too stand in ratios to one another, i.e. one moment can be greater or lesser than another, as Kant knows. ${ }^{32}$ But for Congruence and Steadiness to obtain, all moments of time must stand in the same ratio, viz. unity. If they do not, two durations comprising the same number of moments will not be congruent. Consider two stretches of time LM and NP in the figure below. They are equinumeric-each comprises the same number of moments-but their component moments are unequal. The four moments $d t_{\text {i-1 }}$ making up LM are all in the same ratio to one another, whereas the four moments $d t_{\mathrm{s}-\mathrm{v}}$ in NP stand in unequal ratios. Clearly the two 'determinate times' arising from their respective syntheses, i.e. the very durations LM and NP, are unequal.
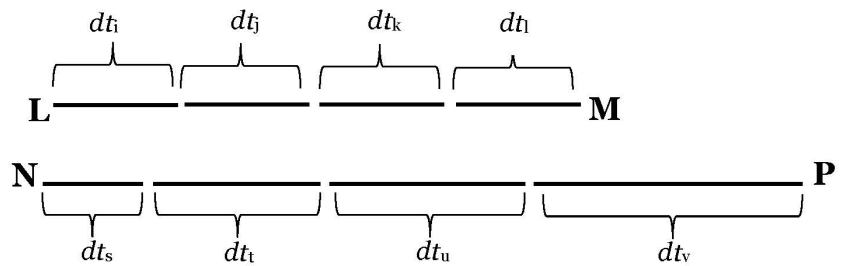

[Fig. 2. Moments stand in ratios. The upper duration LM comprises four equal moments, i.e. whose mutual ratios are unity. The lower time span NP is greater, because its component moments are unequal; specifically, the ratio of a moment to its predecessor is greater than one.]

Then there is no guarantee that any time intervals are congruent, hence that time passes equably. ${ }^{33}$ Newton saw the problem right away, and posited Absolute Time to solve it. But Kant cannot solve it from his resources so far. His categories secure just ratio structure for moments, not the equality of all moment ratios. Then what guarantees that all moments $d t_{\mathrm{i}}$ are equal?

\footnotetext{
${ }^{32}$ Take the 'moment of weight,' i.e. the acceleration $d v$ of terrestrial gravity. It varies by location, as the earth's gravitational potential is a function of distance from the center. E.g., the ratio between the moments of weight at the equator and the North Pole is 9.79/9.81.

${ }^{33}$ Time passes equably just in case all moments of time stand in the same ratio. This ensures that, in our terms, the time function's derivative in respect to an independent variable (say, the position-vector function $\mathbf{x}$ in Cartesian 3-space) is constant, i.e. $d t(\mathbf{x}) d \mathbf{x}=c$.
} 
Inspection in pure intuition cannot do so. Friedman once noted this, in an adjacent context: "How is pure intuition supposedly able to discriminate" equality from small differences in size? How are we "supposed to know, and know a priori," that our intuition has the required structure? The answer is, we cannot. In fact, "the difficulty is a general one," he saw insightfully, because "pure intuition, at least by itself, can in no way demonstrate or exhibit the real possibility" of mere mathematical concepts (Friedman 1990: 218f). For that reason, the equality of moments must be secured by a measure, viz. an assignment of size. Generally, moments qua infinitesimal parts are not pre-equipped with a measure structure. Something else must secure that structure for them. ${ }^{34}$

U-extrinsic groundings of chronometry thereby implode for lack of sufficient structure. The transcendental understanding alone cannot induce metric properties; the topology of time-its uncountable denseness and the ensuing need for a measure-ruins any attempt to do so.

S-extrinsic metrics Another family of construals relies on Kant's thesis that space is the indispensable medium for representing time:

Even inner changes then, for us to make them thinkable, we must make time, as the form of inner sense, graspable figuratively by means of a line. And we make inner change graspable [fasslich] through the drawing of this line (motion). Thereby we can grasp the successive existence of ourselves in different states, by means of outer intuition. (B 292)

This passage allows at least two readings. One is straightforward, though not explicit in scholarship. To represent a duration $\mathrm{D}$, one must produce a segment TR that stands to some other, given segment TU in the same ratio as D stands to a chosen time-unit S. The proportion theory of Euclid's Elements then promises that, for any three magnitudes D, S and TU, a fourth magnitude exists (here, a segment TR) such that $\mathrm{S}: \mathrm{D}:: \mathrm{TU}: \mathrm{TR}{ }^{35}$

\footnotetext{
${ }^{34}$ This inspection can go by two different routes. We could examine any two $d t_{\mathrm{i}}$ to see if they strike the mind as equal; or we could inspect (in inner sense) the passing of time to see if it is uniform. (For Kant and Newton, moments were the 'fluxions' of 'flowing' quantities. Two fluxions are equal if, at their locations, the quantity flows at the same rate.) As I explained above, inspecting inner sense to discover time's rate of passing is a dead end.

${ }^{35}$ See, inter alia, the explications of proportionality and equality of ratios in Michelsen (1791: 175_81), an annotated edition of Euclid's textbook in Kant's time.
} 
Still, Kant's constructivist strictures require that the existence of segment $\mathrm{TR}$, thus of the duration $\mathrm{D}$ that it represents, be proved by straightedge-and-compass construction. But such existence proofs are impossible for 'transcendental' magnitudes, because Euclidean constructions suffer from Sub-coverage (cf. Section 4). Then so do all S-extrinsic accounts of time's metric:

Sub-coverage about time. An infinity of lengths cannot be constructed in pure space. Ergo, an infinity of durations cannot be shown a priori to exist.

And so, S-extrinsic groundings entail that time qua metric manifold is not continuous, but it has an infinity of metric gaps, or non-existent durations (corresponding to 'transcendental' magnitudes). That makes such groundings inadequate.

Friedman's early exegesis implies another reading of Kant's idea above. ${ }^{36}$ According to it, we determine duration by applying the "concept of magnitude to inner sense." But, in the spirit of B 292 above, we "endow time with a metric" indirectly, through the "mediation of spatial intuition." This intuition is really kinematic, as it results from representing the "rectilinear motion," presumably uniform, of a "mathematical point." In turn, intuiting uniform translation is representing a path that lets us "derive the temporal metric from the spatial metric" (Friedman 1990: 242ff). The congruence relations on any such path ground temporal congruence, in that we define equal times to be those in which a point moving uniformly crosses equal distances. More generally, any two durations stand to each other in the same ratio as the distances crossed by a mobile point during those times. ${ }^{37}$ So, time has metric structure in virtue of metric facts about certain privileged space paths.

Unfortunately, this solution is circular, thus fatally flawed. Defined in kinematic terms, motion is uniform if a point crosses equal distances in equal times. So, a notion of temporal congruence is already needed to define 'uniform motion.' Then we cannot use the latter to define equal times from it.

\footnotetext{
${ }^{36}$ Admittedly this account, more implied than asserted, is just one way to read Friedman's explication of B 292 above, I discuss the other in Section 8 below.

${ }^{37}$ Galileo had proved it in Two New Sciences: "if a mobile carried uniformly crosses two distances [spatia], the times of crossing are in the same ratio as the distances" (1638: 151).
} 
At this juncture, the inexorable conclusion is that Transcendental Idealism cannot explain our knowledge of duration. Time as sensible form lacks metric structure; the categories cannot supply it either; and transcendental space is too weak to yield a metric for continuous time. Nil desperandum - an avenue remains open to Kant, but it needs clearing.

\section{Improper solutions, Il: some M-extrinsic metrics}

There is a class of candidates that seem obvious choices for our problem: man-made chronometers, and some celestial body with eminently regular motions. On this construal, time has a metric in virtue of the fact that any two motions of some candidate object are proportional to the times in which they occur.

This idea is easy to dismiss: it fails both Necessity and Coverage. Let $C$ be the material object grounding time's metric. For one, $C$ is contingent, thus so are all the metric facts about it, e.g. the distances it crosses. Then it is equally contingent that time has a metric, depending as it does on $C$ 's existence and behavior: an uncomfortable conclusion. ${ }^{38}$ For another, $C$ must come into being and then cease to be, as all contingent things do. (Even the Sun will die off one day, as Kant freely admitted.) So, there exist times before and after $C$ 's existence, but there is no fact about how long they are, since $C$, their metric ground, is absent ex hypothesi. Thereby, attempts to ground chronometry M-extrinsically in some particular body fall short of Coverage too.

Friedman 1990 implies another M-extrinsic grounding: time has a metric in virtue of the congruence structure of a preferred motion, as Kant had it at B 292. That motion is the path of an inertial, force-free material particle. By the Law of Inertia (a necessary truth, in Foundations) any force-free particle crosses equal distances in equal times. Generally, durations and paths crossed during them are metrically alike: maps from inertial trajectories to their times are isometries.

\footnotetext{
${ }^{38}$ Consider its preposterous consequence: if time must have a metric, and the object $C$ grounds that metric, then $C$ (e.g. the Earth or the Sun) exists necessarily; see Skow 2010.
} 
Still, this too is inadequate. First, inertial translation is the wrong kind of representation: it fails to instantiate alteration, or change of state. Kant wants the pure intuition of "time itself" to represent not just "time as a quantum," or specific durations, but also the concept of causality, as Friedman admits (1990: 243; B 291). But intuiting inertial motion is not representing alteration. Inertial motion is a conserved state-the very opposite of alteration-and Kant knows it well. Simply put, Kant himself warns that not every change of property is as a change of state. Inertial translation typifies that difference: the translating particle changes place without changing its mechanical state. ${ }^{39}$

Second, inertial translation never occurs; Kant's metaphysics entails it cannot obtain. The reason is this. By the Third Analogy, any two bodies are in "community of interaction," bound by 2-way causal bonds. Kant's Third Law then entails all these bonds are grounded in accelerative forces. So, mutual accelerations are universal and metaphysically basic. Hence no single body is causally cut off from its brethren; and their constant action on it amount to deflection from its inertial path, at every instant. Ergo, no actual body ever crosses equal distances in equal times. Then Friedman's M-extrinsic basis for chronometry fails both Objectivity and Measure: representing single bodies in inertial motion is a thought without "objective reality" in actual correlates as its truth-makers; and trying to measure stretches of inertial path (so as to infer durations from their ratios) is doomed to fail.

Objection: this is rash. Even without inertial motion obtaining in fact, the Law of Inertia can be used to ground equality of times, as follows. Let A be a body arbitrarily accelerated. Describe its motion in some inertial frame F, and then in another inertial frame, G. Subtract two velocities, as

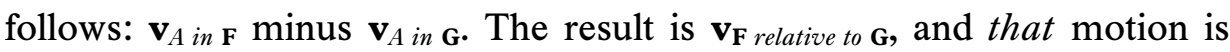
rectilinear uniform (the frames are Galilean); hence it can define equal times, viz. as those in which $\mathbf{G}$ crosses equal distances relative to $\mathbf{F}$. I respond twice. The short answer is, the suggestion cannot be implemented

\footnotetext{
${ }^{39}$ See Foundations (4: 488, 547). In all fairness, Friedman has abandoned this construal now. In Friedman 2003, he still believed that 'time as a pure formal intuition' is the object of Kant's "general doctrine of motion," but he de-emphasized inertial translation as the paradigm intuition instantiating the (schematized) categories.
} 
in Kant's world (or in any classical world with universal gravity, for that matter). In these universes, there is just one genuinely inertial frame defined by matter, namely W. No other true inertial frame is available. So, the procedure just suggested cannot even take off the ground. Determinate answer: the suggestion merely delays the inevitable, it does not remove it. Because all bodies in a Kant-Newton universe are accelerated, all reference frames defined by bodies likewise accelerate, and so are not inertial. At celestial scales, the dominant pattern is mutual orbiting around W. A second inertial frame-as the objection supposes-is thus unavailable.

Part IV Here I present my solution, in Sec. 7; give evidence for it, in Sec. 8; and explain how it works as Kant's absolute time, in Sec. 9.

\section{Chronometry from Kant's 'special metaphysics'}

The most sophisticated construal of Kantian chronometry avoids the interpretive traps I uncovered above. Specifically, Michael Friedman has moved beyond attempts to let the Law of Inertia ground the equality of times. He instead proposes that for Kant it is the categories of substance and interaction that endow time with a ratio structure. They do so via their role as warrant for two principles, viz. Conservation of Mass and of Linear Momentum: "For Kant, [these] laws define what we mean by true temporal uniformity. Two temporal intervals are truly equal, in particular, if they are equal according to these laws" (Friedman 2013: 65; my italics). This turns Friedman's proposal into a species of M-extrinsic grounding. Alas, he offered it merely as a suggestion, and it is not clear how-or even whether-his two invoked principles would cooperate so as to ground chronometry. No matter; I offer here an M-extrinsic solution that is explicit, determinate, and exact. And, it incorporates Friedman's two principles above as subsidiary premises, so it obviates his solution. For my account, I choose a fundamental yet unstated law that is directly entailed by his "special metaphysics of material nature" in Foundations. 
KAL Kant's Area Law: Any volume of matter has a point that, by its radius to the world's mass center, sweeps equal areas in equal times.

To see its real tenor, start with an intuitive precursor, viz. Kepler's second law: relative to the Sun, any solar planet sweeps equal areas in equal times. So, any two areas swept by a planet around the Sun stand in the same ratio as the times it took to cross them. Strictly speaking Kepler's law is false, but something close to it is true. Newton in 1684 proved that a body kept in orbit by a central force around a fixed point covers equal areas in equal times. In the Principia he then showed that Kepler's law is true of any planet, but relative to the mass-center of the solar system, not the Sun (Newton 1713: 375f).

Kant's Area Law “projects into the skies” Newton's result, and makes it universal: true of any piece of matter, not just solar planets; and valid everywhere at cosmic scales, not just in our region. ${ }^{40}$ To grasp the law, take any finite volume of matter $\mathrm{V}$, arbitrarily carved out of the physical universe. Now this volume has a mass center; call it M. Call E the exterior of V: the material volume whose mereological sum with $\mathrm{V}$ gives Kant's Weltganz, the "cosmos, or system of all matter" (4: 562). In turn, E has its own mass center, L. Let $l m$ be the physical line segment with L and M as endpoints; and choose on $l m$ a point $\mathrm{W}$, such that WL is to WM inversely as the mass of $\mathrm{E}$ is to the mass of V. (Intuitively, the larger $\mathrm{E}$ is, the closer $\mathrm{W}$ is to $\mathrm{L}$, and vice versa.) Evidently, $\mathrm{W}$ is the mass-center of the aggregate volume $\mathrm{V}+\mathrm{E}$. Kant called that point the "common center of gravity of all matter" (4: 563). The law now simply states:

KAL Relative to the cosmic center $\mathrm{W}$, any mass-center M sweeps equal areas in equal times. Generally, areas swept by MW are always in exact proportion to the times it takes MW to sweep them.

Recall that the material volume $\mathrm{V}$ was chosen arbitrarily, hence KAL holds of any mass center as it moves relative to $\mathrm{W}$. The law is truly general, though it is about relative to a preferred point $\mathrm{W}$, the cosmic mass center.

\footnotetext{
${ }^{40}$ The phrase is Friedman's, who coined it for Kant's extending to cosmic scales Newton's local principle of inferring inertial mass from terrestrial weight (2013: 304). Friedman's imagery of celestial projection is apt, so I borrow it to denote a similar Kantian extension.
} 


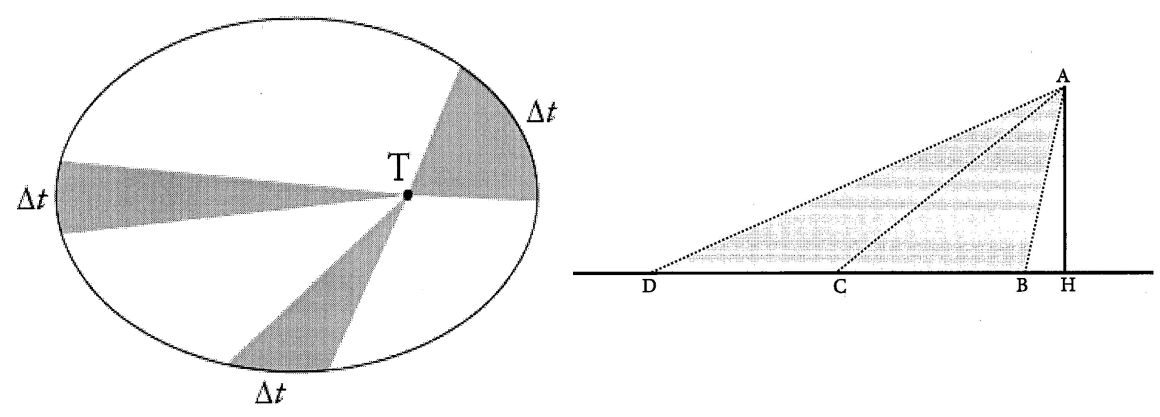

[Fig. 3. Areal motion around W. (a) Kepler orbits obey the area law; the grey sectors are equal areas. (b) Inertial translation also conforms to the area law. AH is the common height of the two triangular areas swept during equal times, relative to $O$. By the law of inertia, $B C$ and $C D$ are equal distances.]

The motion of any $\mathrm{M}$ around the center $\mathrm{W}$ is a so-called Kepler orbit, or conic section: a hyperbola, parabola, ellipse or circle (Fig. 2a). Such orbits are special for our purposes, because any object in a Kepler orbit sweeps equal areas in equal times. Moreover, KAL contains Friedman's choice as a degenerate case. If inertial translation could occur, it too would obey Kant's Area Law (see Fig. 2b). This speaks in favor of my solution.

Crucially, my solution entails Congruence and Steadiness, the grounding corollaries of chronometry. To show that, first I introduce a technical notion. Let $\mathrm{V}$ be a volume of unit mass, $\mathrm{M}$ its mass center, $r$ the distance from $\mathrm{M}$ to the cosmic center $\mathrm{W}$, and $d s$ the infinitesimal path that $\mathrm{M}$ crosses in a moment of time $d t$. Define $\mathbf{H}$ as the cross product $r \times$ $(d s / d t) .{ }^{41}$ Next I invoke two of Kant's tenets.

Conservation: "the total quantity of matter remains the same throughout changes in corporeal nature" (4: 541). So, in any given volume, mass is a conserved scalar. Space: any two material volumes stand in determinate distance relations, because space is the form of outer sense.

Conservation is a basic principle of his metaphysics, and Space a result of the Transcendental Aesthetic. Jointly they entail a very valuable result: for

\footnotetext{
${ }^{41}$ Geometrically interpreted (as it was in Kant's time), a cross product (of two directed quantities) is an area. Note that $d s / d t$ is M's instantaneous velocity, and so $\mathbf{H}$ is the angular momentum of the volume $\mathrm{V}$ relative to $\mathrm{W}$.
} 
any proper part of the material world, the quantity $\mathbf{H}$ is well defined at all times.

Now let A and B be two arbitrary points on the orbit of any masscenter $\mathrm{M}$ in motion relative to $\mathrm{W}$, and let $t_{a}$ and $t_{b}$ be the times at which $\mathrm{M}$ crosses A and B, respectively. Stated precisely, Kant's Area Law is: ${ }^{42}$

$$
\int_{t a}^{t b} d t=k_{V} \int_{A}^{B} \mathbf{H} d s
$$

In words, the length of time it takes $M$ to cross from $A$ to $B$ is proportional to the area $\mathbf{H}$ it sweeps around W. Then KAL entails Congruence as a trivial corollary, due to Conservation and to equality being symmetric and transitive:

$$
\int_{t a}^{t b} d t=k_{V} \int_{A}^{B} \mathbf{H} d s=k_{V} \int_{C}^{D} \mathbf{H} d s=\int_{t c}{ }^{t} d t
$$

Ergo, whenever two $\mathrm{W}$-areas are equal, their respective times are too. Steadiness follows as well, but it requires an extra assumption, which I supply in Section 9. For now, I just state it:

$$
d \mathbf{H} / d t=0
$$

The expression above conveys a universal feature of Kantian matter: it moves around the world's center at a strictly constant areal rate. So, it is metaphysically impossible to slow down or speed up that motion. But those areas - their ratios-are the very ground of time's own rate of passing. It follows that time too "passes equably" at a rate that "cannot change," just as Newton required. Ergo, in Kant's system Steadiness is a metaphysical fact, and so nothing can change the passing of time.

The quantity $\mathbf{H}$ solves the problem that eluded Longuenesse and others-for it gives a preferred measure for Kant time, i.e. for the size of single time-moments $d t$. With $\mathbf{H}$ as their grounding measure and KAL securing their isomorphism to $\mathbf{H}$, all moments have the same size, and integrat-

\footnotetext{
${ }^{42}$ In the expression (8.1), $k_{V}$ is a system-bound constant, viz. the mass of V. Recall that $\mathbf{H}$ was defined for a system of unit mass.
} 
ing over the same number of moments yields equal times, or durations of the same length. ${ }^{43}$

\section{Evidence in context}

Kant's Area Law is as true as his metaphysics of matter, because it follows deductively from it. I give a proof sketch below, and I develop it in Appendix I; the proof has three steps.

First, I establish a key lemma. To emulate Kant, I call it the 'Law of Antagonism for Rotation,' or LAR: relative to the world-center W, for any torque $\mathbf{t}$ on a mass $\mathrm{V}$, there is an equal and opposite torque $-\mathbf{t}$ induced by $\mathrm{V}$ on the source of $\mathbf{t}$. LAR secures inertia of rotation, i.e. that the cosmos does not self-accelerate around axes of spin through W.

Second, from Kant's metaphysics I derive another law, Isochronism: if a particle sweeps equal areas, it takes equal times to do so. This law is true in any inertial frame whatsoever. To establish Isochronism, I reverseengineer Newton's strategy for proving Kepler's second law (1713: 34ff).

Third and last, I extrapolate Isochronism to the only inertial frame obtaining in Kant's world, namely the frame with the origin at $\mathrm{W}$; he called it "absolute space." ${ }^{44}$ So any system of bodies sweeps equal areas in equal times, relative to W. That is the real content of Kant's Area Law, which I just established. But my proof-by-extrapolation requires three conditions so as to be sound:

1. The cosmic center $\mathrm{W}$ does not accelerate.

2. All areas swept by MW lie in the same plane.

3. The net force on $\mathrm{M}$ is along the line MW.

Kant is able to defend them all, but the third comes at a price. He justifies (1) above on epistemic grounds: accelerations on W are not possible objects of experience. ${ }^{45}$ Assumption (2) is a trivial corollary of LAR above. If

\footnotetext{
${ }^{43}$ In contrast, it is unclear whether Kant could obtain a notion of equal temporal distance. Anyway, with my solution in place, he does not really need that notion any more.

${ }^{44}$ For the "explicit connection" between this term and the mass-center of a system of interacting bodies, see Friedman 2013: 503ff.

${ }^{45}$ For Kant all knowable motions are kinematic relations between material entities. Hence for W's accelerations to be knowable, they must be relative to a material frame external to
} 
all mutual torques relative to $\mathrm{W}$ are pairwise balanced, it follows that any mass-center $\mathrm{M}$ orbits around an axis of rotation (through $\mathrm{W}$ ) that remains fixed, hence the orbit is always in the same plane. Note that (1) and (2) together entail that $\mathrm{W}$ is the origin of a true inertial frame, non-rotating and unaccelerated. This result is absolutely crucial for my proof; without it, I have no right to extend the Kepler law to W. Finally, (3) falls out of Proposition 5 of Dynamics, the heart of Kant's metaphysics of matter: "an attractive force is the second essential basic force of matter" (4: 508). I explain it below.

Here too I have avoided anachronism, for Kant's age already had a general law of isochronous area motion: Conservation of Angular Momentum. But, this principle was a posteriori. Theorists then either asserted it blankly or accepted it because it entails equations of motion for mechanics, and those were empirically true. ${ }^{46}$ Being a posteriori, Conservation of Angular Momentum lacks necessity, so it cannot be a necessary ground for time's metric. Only KAL is that ground, precisely because it is a priori. Still, KAL is just the conservation law above, though restricted to the world center $\mathrm{W}$.

\section{Material conditions for possible temporal experience}

My solution puts Kant in the tradition of philosophers, from Aristotle to Leibniz, for which time is the measure of change: of motion as "basic determination of anything that is to be an object of outer senses" (4: 476).

However, all motion in the world is always irregular, which conflicts with the widely held idea that time's passing is uniform. Path motion is provably irregular: in equal times, a particle crosses unequal lengths. ${ }^{47} \mathrm{Ar}$ eal motion is also irregular, except relative to W. In respect to any other

the physical world. Ex suppositione, no such frame is givable to experience. So, "absolute motion," i.e. not relative to a material frame, "is utterly impossible" (4: 563).

${ }^{46}$ A case of blunt assertion is Daniel Bernoulli (1746: 59). For hypothetico-deductive defenses of Conservation of Angular Momentum (via entailed equations of motion) see Euler (1776: §29) and Lagrange (1788: Part II, § iii.2), who called it the 'Principle of Areas.'

${ }^{47}$ This is evident. The path length is the time-integral of the force $\mathbf{f}$ on the particle, viz. $\int$ $\mathbf{f}(x) d t$. Forces are variable-their strength differs at different locations $x$; then so is their integral. 
point $\mathrm{C}$, the Area Law fails. This failure is called precession, and it entails that in equal times a body sweeps unequal areas relative to $C$ (see fig. 4).
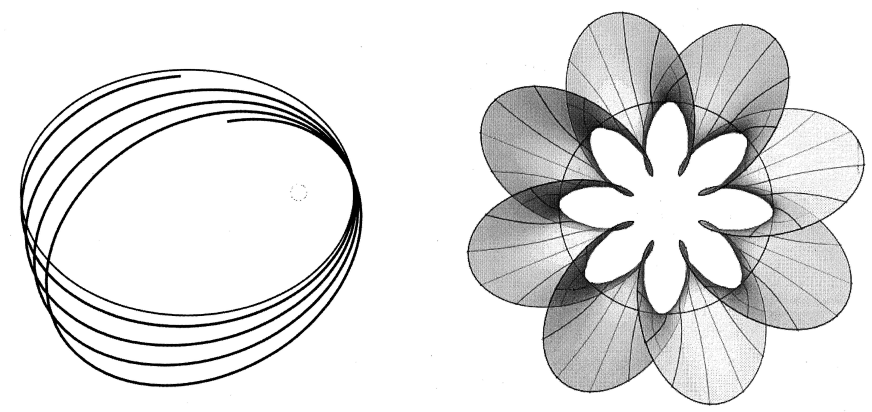

[Fig. 4. Two failures of the Area Law. On the left is a Kepler orbit that precesses, viz. its containing plane rotates around an axis passing through its focus. As a result, the orbit fails to close in on itself, and areas swept in equal times are not equal. On the right, the orbit's plane itself moves in a circle around a center. The shaded grey surface is the area swept by the particle around the orbiting focus of its own orbit.]

Ergo, nothing but $\mathrm{W}$ makes areal motion truly isochronous (as KAL states) and so my solution is uniquely adequate: it alone can ground time's metric for Kant, and vindicate Congruence and Steadiness for him.

Point $\mathrm{W}$ has been explicated as the origin of a preferred material frame of reference, which Kant named "absolute space." He privileged it because it "unites all appearances" of motion: it yields a unified description for the true motions of all bodies, whereas their merely apparent motions lack unity, each being relative to some particular, arbitrary frame. To reach Kant's absolute space, we must first locate $\mathrm{W}$, its origin. But $\mathrm{W}$ is not given, so we must approximate it by a series of successively located mass-centers. ${ }^{48}$ This makes W a "kind of limit," namely of mass-centers

\footnotetext{
${ }^{48}$ The procedure in a nutshell is this. Take the earth's volume E and (by applying the Newton-Kant dynamical laws) determine the net force $\mathbf{f}_{\mathrm{E}}$ on the earth's mass center. Now move up to $\mathrm{E}_{2}$, a larger volume containing the earth and the masses responsible for the force $\mathbf{f}_{\mathrm{E}}$ on it. Locate the mass center of $\mathrm{E}_{2}$, then infer to the net force on it. Repeat, ad libitum. Any further iteration is a closer approximation of areas swept around W, which areas (by KAL) are isomorphic to their times. For complete details, see Friedman (2013: 474-509).
} 
for a "sequence of ever more comprehensive" material volumes. If $\mathrm{W}$ is the limit of a series of law-based iterations, then so is $\mathrm{W}$-areal motion, which grounds time's metric. It follows that, to represent objectively the notions of equal times and uniform time flow, we must adopt "absolute time" as a regulative principle: Regard all areal changes as provisional measures of time; and endeavor to get ever closer to locating $W$, and thereby to areas swept relative to it. My construal thus makes absolute time the full analogue of Kant's absolute space. As regulative ideal, the latter demands that we "always pursue the sequence one step further while never accepting any given finite stage as terminal" (Friedman 2013: 500, 506).

Material conditions of possibility. In Kant's system time has a metric only because of KAL, not intrinsically. But the law depends on two facts:

W-rest: the cosmic mass-center W does not accelerate. Centrality: the nature of matter includes a central attractive force.

W-rest is really two claims: $i$ ) the center W does not accelerate; and $i$ ) any axis of revolution passing through $\mathrm{W}$ remains self-parallel: it does not change direction over time. I prove both in the Appendix. Centrality is the metaphysical claim that any two substances exert an interaction-at-adistance, causing accelerations along the straight line between their masscenters.

This claim is absolutely crucial to solving Kant's predicament. Centrality is sine qua non for his metaphysics to entail his Area Law, and thereby for KAL to be synthetic a priori. In turn, this epistemic status secures $n e-$ cessity for his law, thus for the chronometric structure it underwrites.

And yet, Centrality could have failed to obtain, for three reasons. First, it is non-trivial: forces between masses could well be different (e.g. they all could be non-central or contact interactions) and then KAL would not be a fact. ${ }^{49}$ Second, it must be valid for all masses and at all scales, or else ascending to celestial levels (where areal motion ought to approximate orbiting around $\mathrm{W}$ ) will do nothing to get us closer to measuring absolute time. Third, Centrality is no direct outcome of applying the Critique to

\footnotetext{
${ }^{49}$ Contact forces are non-central by definition (in general, they do not act on the line of centers between bodies). And, some action-at-a-distance forces (e.g., magnetic inductions) act perpendicularly to the line of centers, not along it.
} 
the concept of matter, which is Kant's official program in Foundations. Rather, it is a corollary of his Balancing Argument, which is premised on non-trivial facts about the nature of matter. ${ }^{50}$ It is to Kant's credit that he crafted his metaphysics so that KAL obtains.

But if Centrality is sine qua non for time to have a metric then his metaphysics of matter is indispensable to the Critical project of explaining the possibility of experience. Bluntly put, without Centrality, KAL fails; and if the law fails, time has no metric; if time is metrically amorphous, knowledge of duration is just a chimera. So, the possibility of temporal experience requires a unique metaphysics, viz. Kant's doctrine of material nature. That makes attractive force a "material condition of possibility," as he put it in the 1790s. In retrospect, transcendental idealism was always incomplete.

Adequacy. My Kantian grounding of time's metric meets the constraints on solutions I listed in Sec. 3 above. Necessity. Kant's Area Law follows deductively from his commitments in Foundations; so it carries the necessity he believed they have. However, this latter aspect deserves extensive discussion that I cannot provide here. For instance, my proof of the lemma LAR mimics Kant's proof of the Equality of Action and Reaction. But his proof relies on epistemic necessity, while his laws of motion are metaphysically necessary, grounded in the nature of matter. Coverage. The key to securing coverage for all of time is Kant's metaphysical law, Conservation. It entails two things: the cosmic mass-center $\mathrm{W}$ exists as long as matter exists; and for any cosmos-part there exists a point $\mathrm{M}$ coeval with it. Underlying every causal process is the fact that some $\mathrm{M}$ changes position relative to $\mathrm{W}$, thus describing an area. These areas ground the temporal lengths of physical changes supervening upon them. Conversely, there is no event for which there is no MW-swept area to underwrite its duration. Moreover, my solution escapes the threat of Sub-coverage, for it does not require Kant to exhibit a priori the existence of any specific duration. So, it avoids the straightedge-and-compass con-

\footnotetext{
${ }^{50}$ Cf. 4: 508 , Proposition 5 of Dynamics. Kant proves that attraction is essential to matter by a 'balancing argument.' He starts with the fact of impenetrability, argues that it must be caused by a repulsive force, then proves that an attractive force-qua direct and central actio in distans - must exist to balance that repulsion, i.e. to keep matter into structures stable over time. For an excellent account, see Smith 2013.
} 
straint on constructions in pure intuition. That any duration exists is given by metaphysical facts about matter and its nomic behavior. But these facts need not be represented as Eudoxean ratios of Euclidean incommensurables; they can be treated by algebraic formalism, in tune with the arithmetizing spirit of Kant's time. Exactness. Start with an arbitrary volume and carry out the approximating procedure for locating mass-center frames that Friedman outlined on Kant's behalf (2013: 474-509). Any iteration of the procedure gets us closer to $\mathrm{W}$, the privileged world center. Thus, by successively applying his metaphysical laws of motion, we get arbitrarily close to measuring areas swept relative to W. By Kant's Area Law, ratios of these areas ground time measurements to any degree of exactness sought.

\section{Conclusions: fallout}

I have explored Kant's options for grounding time's metric, in the wake of his denial of the Newton-Euler thesis that time is immaterial and nonmental. The strong temptation is to suppose that some structure in the mental machinery of the Critique can ground the metric properties of time. That hope is in vain, I have explained. Were time discrete, one Kantian form-the categories of quantity-would be enough to induce metric structure on time as we know it. But time is continuous, and so the number-structure of the Transcendental Analytic is not strong enough to determine it quantitatively.

For that reason, I have argued, Kant must rely on his metaphysics of matter. In particular, his doctrine entails a synthetic a priori truth I have called Kant's Area Law. It guarantees that certain universal patterns of motion-areas swept relative to the cosmic mass center-always stand to each other in the same ratios as the times required for those motions. These areas then, by virtue of their nomic relations, can ground time's metric and also guarantee the necessity needed for chronometric structure in Kant's overall doctrine.

At the same time, my result reverberates through his system, and gives us a reason to look afresh at some thorny problems of interpreta- 
tion. One implication regards the notoriously obscure relation between the metaphysics of Foundations and the transcendental account in the Critique. My chronometric grounding implies that we must see the two Kantian doctrines as inextricably related, such that the metaphysics is indispensable to that account, not just an epitome of it. In sum, time in the Critique has no metric aspects, and so the Transcendental Aesthetic and the Schematism (of the quantity categories) are essentially incomplete. KAL does induce metric structure on time, but it depends on the metaphysics of matter defended in Foundations, because of Centrality. Without his matter theory, important aspects of experience would remain unaccounted for by transcendental idealism, despite Kant's official claims to completeness of explanation. That is just as well; as if to sanction my point, in Opus postumum Kant moved to expand his doctrine with a "material condition" for the possibility of experience. ${ }^{51}$ The post-Critical Kant conceded that his Critique needed a material complement after all; which reinforces my overall case in this paper.

Another implication concerns the status of Kant's matter theory. Though in Foundations he submits that his doctrine-including the key claim that attractive force is essential to matter, ergo Centrality obtains-is synthetic a priori, Kant mood there is hesitant. For he suggests that his 'Dynamics' (where he asserts Centrality) is hypothetical or heuristic, a mere candidate account in competition with the "system of absolute impenetrability." In light of my result, Kant must not waver any longer. His material metaphysics can ground time's metric, whereas "absolute impenetrability" cannot, as it lacks the key ingredient, viz. action-at-a-distance attractive force. $^{52}$

Incidentally, Kant's situation presents early modern thought with a stark dilemma: accept Newton's transcendentally-real Absolute Time on pain of their doctrines being incomplete; or adopt Kant's immanent metaphysics of material substance qua active at a distance, which is the sole extrinsic source of chronometric structure. Tertium non datur.

\footnotetext{
${ }^{51}$ That material condition was the 'ether', an all-pervading, quasi-material medium needed to carry interaction forces across space; for lucid details, see Förster 2000: 75-115.

${ }^{52}$ Kant means a matter theory in which the basic object is the rigid body, and all interactions are mediated by contact forces; for detailed explanations, see Friedman 2013: 111-17.
} 


\section{Appendix: proof of Kant's Area Law}

First I prove the lemma LAR. Then from Kantian premises I prove Kepler's second law, relative to arbitrary inertial frames. Finally, I use LAR to show that Kepler's result holds relative to W, just as Kant's Area Law states.

Step I. The Law of Antagonism for Rotation, or LAR, is: For any torque $\mathbf{t}$ on a mass $V$ relative to the world-center, there is an equal and opposite torque - $\mathbf{t}$ induced by $V$ on the source of $\mathbf{t}$. Torque is the other ultimate kind of causal agency in the metaphysics of Foundations. Kant left torque unthematized, but his doctrine supports it easily, for my purposes here $^{53}$

To derive LAR, I rely on Kant's resources and proof-strategy; specifically, on his basic principle of Phenomenology: "matter can only be thought to move or rest relative to matter... Hence, absolute motion (i.e. motion thought without any relation of matter to other matter) is simply impossible." ${ }^{54}$ Ergo, all facts about (objective) orbits are motions relative to material frames external to the system in orbit. That includes angular accelerations, i.e. changes in the length and direction of the axis of revolution.

My proof of LAR is by reductio, just as Kant proved the Equality of Action and Reaction; LAR is really just the rotational analogue of Kant's law. Suppose the torque $\mathbf{t}$ on $\mathrm{V}$ is not equal and opposite to V's torque $\mathbf{t}^{\prime}$ on its exterior $\mathrm{E}$. Then a net torque $\mathbf{w}$ results, acting on the axis $w z$ passing through the cosmic mass-center W. By Kant's principle above, for this ensuing fact to be knowable it must consist in a kinematic change of the

\footnotetext{
${ }^{53}$ The other is "moving forces," causing straight-line motion. Torque is analogous to force; it causes bodies in circular motion to speed up or slow down around the axis of spin.

${ }^{54}$ See 4: 559, General Remark. Kant's Phenomenology is a philosophical explication of true motion (qua universal feature of body), i.e. of kinematic quantities counting as objective for all possible observers.
} 
axis $w z$ relative to a material frame external to the world-whole. By definition no such frame is available, hence a motion relative to $i t$ is impossible. So, denying LAR entails the obtaining of an impossible, "absolute" motion; then LAR must be apodictically true, on pain of contradiction:

any proof of a law of motion amounting to a demonstration that the law's opposite would entail a motion of the entire cosmic system is an apodictic proof of its truth. That is simply because then absolute motion would follow, which is wholly impossible... Such is the law of antagonism in all community of matter through motion. (4: 562f.)

LAR too governs a type of 'antagonism,' or balanced interaction by accelerative causes: via torques as causes of angular accelerations relative to $\mathrm{W}$.

Step II. Now I move to prove Isochronism. I rely on three premises from Kant's metaphysics: the Parallelogram of Motions, the law of inertia, and Centrality, i.e. the central force of attraction on any material particle..$^{55}$ This step requires reasoning on a diagram; see Fig. 8 .

${ }^{55}$ For Kant's Parallelogram Law, cf. Friedman (2013: 379); for his Law of Inertia, 4: 543f. 


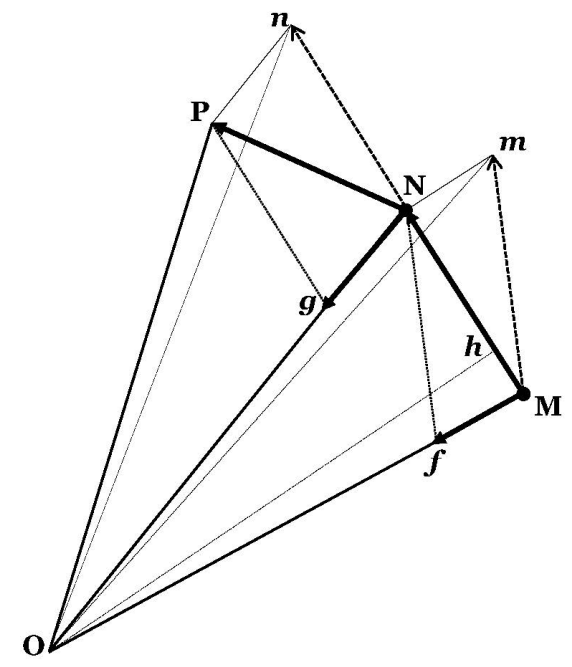

[Fig. 5. Areal motion around a fixed center relative to an inertial frame. MON and Non are infinitesimal triangular areas swept by a particle at $M$ in two consecutive instants.]

Let a particle $\mathbf{p}$ of unit mass be at point $\mathrm{M}$, moving with velocity $\mathrm{M} m$. The attractive force causes on $\mathbf{p}$ an acceleration $\mathrm{M} f$ directed toward $\mathrm{O}$, a fixed center. This is Centrality. Namely, the force on $\mathbf{p}$ acts on the line between $\mathbf{p}$ and the mass-center of whatever exerts the force on it. Only then may we regard $\mathrm{O}$ as a fixed center of force.

By the Parallelogram Rule, the resulting motion is MN. That is p's $a c$ tual trajectory during an instant $d t_{1}$. At the end of that instant, $\mathbf{p}$ has arrived at $\mathrm{N}$. By the law of inertia, $\mathbf{p}$ would continue with its current velocity $\mathrm{N} n$. However, at $\mathrm{N}$ the force of attraction again impresses on $\mathbf{p}$ an acceleration $\mathrm{Ng}$ toward $\mathrm{O}$. And the Parallelogram Rule again entails that, over the current instant $d t_{2}$, the particle's actual path is NP. Three triangular areas arise. I call $\mathbf{A}$ the area $\mathrm{MNO}, \mathbf{B}$ the area $\mathrm{N} n \mathrm{o}$, and let $\mathbf{C}$ denote NPO. All are infinitesimal surfaces. Finally, let $\mathrm{OH}$ be the joint height of two triangles, $\mathrm{MNO}$ and $\mathrm{N} n \mathrm{O}$.

I take as given $\mathbf{A}=\mathbf{C}$, namely, the antecedent of Isochronism. My task is to prove that the two consecutive instants $d t_{1}$ and $d t_{2}$ are equal. But that 
is equivalent to proving that $\mathrm{MN}$ and $\mathrm{N} n$ are congruent. Ipso facto, showing $d t_{1}=d t_{2}$ amounts to proving Steadiness. ${ }^{56} \mathrm{I}$ begin by proving $\mathbf{B}=\mathbf{C}$. Recall that a triangle's area equals half the base multiplied by the respective height. Notice that $\mathbf{B}$ and $\mathbf{C}$ have a common base NO. And their respective heights are equal too, as perpendiculars between parallels. ${ }^{57}$ Hence, $\mathbf{B}=\mathbf{C}$, and $\mathbf{A}=\mathbf{C}$ per hypothesin. Ergo, $\mathbf{A}=\mathbf{B}$. Notice now that the triangles $\mathrm{MON}$ and Non, whose areas they are, have a common height, $\mathrm{OH}$. It follows that their respective bases are congruent. That is, $\mathrm{MN}=\mathrm{N} n$, and so $d t_{1}=d t_{2}$.

Step III. Kant's Area Law results from extrapolating the result in Step II, as follows. Substitute the world center $\mathrm{W}$ for the fixed point $\mathrm{O}$ above; and the point $\mathrm{M}$ for the particle $\mathbf{p}$. It follows that, relative to the cosmic center, any mass center $M$ sweeps equal areas in equal times. QED.

\section{Bibliography}

Agnesi, M.G. 1748. Instituzioni analitiche ad uso della gioventú italiana, vol. II. Milan.

Alexander, H.G. (ed.) 1970. The Leibniz-Clarke Correspondence. Manchester UP. Bernoulli, D. 1746. Nouveau problème de mécanique. Mémoires de l'académie des sciences 1: 54-70. Berlin.

Bricker, Ph. 1993. Intrinsic vs. Extrinsic Distance Relations. Midwest Studies in Philosophy 18, eds. S. French et al., 271-94. University of Notre Dame Press.

Cantor, G. 1874. Über eine Eigenschaft des Inbegriffes aller reellen algebraischen Zahlen. Journal für reinen und angewandten Mathematik 77: 258-262.

Cousin, J.A. 1777. Leçons de calcul differentiel et de calcul integral, vol. I. Paris. De Risi, 2007. Geometry and Monadology. Basel: Birkhäuser.

[du Châtelet, E.] 1740. Institutions de physique. Paris.

56 My warrant is Kant's explanation that we represent time by a line (B 292). Hence, infinitesimal segments can stand for instants. My task is to prove that all such segments are congruent.

${ }^{57}$ By construction, $\mathrm{M} m \mathrm{~N} f$ and $\mathrm{N} n \mathrm{P} g$ are parallelograms. 
Dunlop, K. 2009. The Unity of Time's Measure: Kant's Reply to Locke. Philosophers' Imprint 9 (4): 1-31.

Euler, L. 1736. Solutio problematis ad geometriam situs pertinentis. Commentarii academiae scientiarum Petropolitanae 8: 128-140.

- . 1768. Institutiones calculi integralis, vol. I. St Petersburg.

Falkenstein, L. 1995. Kant's Intuitionism. University of Toronto Press.

Förster, E. 2000. Kant's Final Synthesis. Harvard UP.

Friedman, M. 1983. Foundations of Space-Time Theories. Princeton UP.

- - . 1990. Kant on Concepts and Intuitions in the Mathematical Sciences. Synthese 84: 213-257.

- . 2003. Transcendental Philosophy and Mathematical Physics. Studies in History and Philosophy of Science 34: 29-43.

- - . 2013. Kant's Construction of Nature. Cambridge UP.

Galilei, G. 1638. Discorsi e dimostrazioni matematiche intorno à due nuove scienze. Leyden: Elsevier.

Gerhardt, C.I. (ed.) (1849-). Leibnizens mathematische Schriften, vol. II. Berlin.

Glymour, C. 1972. Physics by Convention. Philosophy of Science 39: 322-340.

Grabiner, J. 2011. The Origins of Cauchy's Rigorous Calculus. Dover.

Grünbaum, A. 1970. Space, Time and Falsifiability. Philosophy of Science 37: 469-588.

Hindenburg, C.F. 1781. Novi systematis permutationum, combinationum ac variationum primae lineae. Leipzig.

Hyder, D. 2013. Time, Norms, and Structure in Nineteenth-Century Philosophy of Science. The Oxford Handbook of the History of Analytic Philosophy, ed. M. Beaney, 250-279. Oxford UP.

Kant, I. 1794. Metaphysische Anfangsgründe der Naturwissenschaft. Frankfurt.

-_- 1902- . Gesammelte Schriften, ed. Prussian Academy of Sciences. Berlin: Reimer.

Kemp Smith, N. 1967. A Commentary to Kant's Critique of Pure Reason. Humanities Press.

Lacroix, S.F. 1796. Traité du calcul différentiel et du calcul intégral, tome II. Paris.

Lagrange, J.L. 1788. Méchanique analitique. Paris. 
Lambert, J. 1768. Mémoire sur quelques propriétés remarquables des quantités transcendantes circulaires et logarithmiques. Histoire de l'Academie Royale de Sciences et Belles Lettres de Berlin, Année 1761: 265-322.

Lebesgue, H. 1927. Sur le développement de la notion d'intégrale. Revue de Métaphysique et de Morale 34: 149-167.

LeSeur, Th. \& Fr. Jacquier. 1768. Elemens du calcul integral. Parma.

Liouville, J. 1844. Sur des classes très étendues de quantités dont la valeur n'est ni algébrique, ni même reductible à des irationelles algébriques. Comptes Rendus de l'Académie des Sciences de Paris 18: 883-85.

Longuenesse, B. 1998. Kant and the Capacity to Judge, trans. Ch. Wolfe. Princeton UP.

Massey, G. 1970. Is 'Congruence' a Peculiar Predicate? PSA: Proceedings of the Meeting of the Philosophy of Science Association, 606-615. Springer.

Massimi, M. 2016. Grounds, Modality, and Nomic Necessity in the Critical Kant. Kant and the Laws of Nature, eds. M. Massimi and A. Breitenbach, 150-170. Cambridge UP.

Maudlin, T. 2007. The Metaphysics within Physics. Oxford UP.

Messina, J. 2016. Kant's Necessitation Account and the Nature of Natures. Kant and the Laws of Nature, 131-149.

Michelsen, J.A.Chr. 1791. Euclides Elemente. Berlin.

Newton, I. 1713. Philosophiae Naturalis Principia Mathematica, ed. R. Cotes. London.

Sauri, Abbé. 1778. Cours de mathematiques, vol. IV. Paris.

Schultz, J. 1784. Erläuterungen über des Herrn Professor Kant Critik der reinen Vernunft. Leipzig: Dengel.

Skow, B. 2010. Extrinsic Temporal Metrics. Oxford Studies in Metaphysics, ed. D. Zimmerman, vol. 5, 179-202. Oxford UP.

- - . 2011. On the Meaning of the Question "How Fast Does Time Pass?" Philosophical Studies 155: 325-344.

Smit, H. 2009. Kant on Apriority and the Spontaneity of Cognition. Metaphysics and the Good, eds. S. Newlands and L. Jorgensen, 189-251. Oxford UP.

Smith, S. 2013. Does Kant have a pre-Newtonian picture of force in the balance argument? Studies in History and Philosophy of Science 44: 470-480.

Stang, N. 2016. Kant's Modal Metaphysics. Oxford UP. 
Sutherland, D. 2004. Kant's Philosophy of Mathematics and the Greek Mathematical Tradition. Philosophical Review 113: 157-201.

- - . 2005. The Point of Kant's Axioms of Intuition. Pacific Philosophical Quarterly 86: 135-159.

- - . 2014. Kant on the Construction and Composition of Motion in the Phoronomy. Canadian Journal of Philosophy 44: 686-718.

Valaris, M. 2008. Inner Sense, Self-Affection, and Temporal Consciousness in Kant's Critique of Pure Reason. Philosophers' Imprint 8 (4): 1-18.

Van Cleve, J. 1999. Problems from Kant. Oxford UP.

Van Fraassen, B. 1969. On Massey's Explication of Grünbaum's Conception of Metric. Philosophy of Science 36: 346-353.

Wantzel, M.L. 1837. Recherches sur les moyens de reconnaître si un problème de Géométrie peut se résoudre avec la règle et le compas. Journal de mathématiques pures et appliquées 2: 366-372.

Watkins, E. 1998. The Argumentative Structure of Kant's Metaphysical Foundations of Natural Science. Journal of the History of Philosophy 36: 567-593. 